\title{
Uma Solução Híbrida Analítico-Numérica para o Escoamento Laminar em Dutos Bicônicos
}

\section{A Hybrid Analytical-Numerical Solution to the Laminar Flow inside Biconical Ducts}

\author{
Thiago Antonini Alves'; Ricardo Alan Verdú Ramos²; Cassio Roberto Macedo Maia ${ }^{3}$
}

\section{Resumo}

Neste trabalho foi apresentada uma solução híbrida analítico-numérica do problema fluidodinâmico de escoamento laminar desenvolvido de fluidos Newtonianos no interior de dutos bicônicos utilizando a Técnica da Transformada Integral Generalizada - TTIG. Para facilitar o tratamento analítico e a aplicação das condições de contorno uma Transformação Conforme foi utilizada visando transformar o domínio em um sistema de coordenadas mais apropriado. Feito isso, a TTIG foi aplicada na equação do momentum para determinação do campo de velocidade. Os resultados numéricos foram obtidos para parâmetros fluidodinâmicos de interesse, tais como: velocidades média e máxima, fator de atrito de Fanning, número de Poiseuille, fator de Hagenbach e comprimento de entrada fluidodinâmico.

Palavras-chave: Transformada Integral. Transformação Conforme. Escoamento Laminar. Bicônico.

\begin{abstract}
In this work was presented a hybrid analytical-numerical solution to hydrodynamic problem of fully developed Newtonian laminar flow inside biconical ducts employing the Generalized Integral Transform Technique (GITT). In order to facilitate the analytical treatment and the application of the boundary conditions, a Conformal Transform was used to change the domain into a more suitable coordinate system. Thereafter, the GITT was applied on the momentum equation to obtain the velocity field. Numerical results were obtained for quantities of practical interest, such as maximum and minimum velocity, Fanning friction factor, Poiseuille number, Hagenbach factor and hydrodynamic entry length.
\end{abstract}

Keywords: Integral Transform. Conformal Transform. Laminar Flow. Biconical.

\footnotetext{
${ }^{1}$ Doutor em Engenharia Mecânica, Departamento Acadêmico de Mecânica, Universidade Tecnológica Federal do Paraná/Campus Ponta Grossa - DAMEC/UTFPR/Ponta Grossa; thiagoaalves@utfpr.edu.br

${ }^{2}$ Doutor em Engenharia Mecânica, Departamento de Engenharia Mecânica, Faculdade de Engenharia de Ilha Solteira, Universidade Estadual Paulista "Júlio de Mesquita Filho" - DEM/FE/IS/Unesp; ramos@dem.feis.unesp.br

${ }^{3}$ Doutor em Engenharia Mecânica, Departamento de Engenharia Mecânica, Faculdade de Engenharia de Ilha Solteira, Universidade Estadual Paulista "Júlio de Mesquita Filho"- DEM/FE/IS/Unesp; cassio@dem.feis.unesp.br
} 


\section{Lista de Símbolos}

$A_{i} \quad$ coeficiente do termo transformado

$A_{S}$ área da seção transversal do duto $\left[\mathrm{m}^{2}\right]$

$B_{i j}$ coeficiente do termo transformado

$D_{h}$ diâmetro hidráulico [m]

$f \quad$ fator de atrito de Fanning

$h_{u}$ coeficiente métrico do sistema de coordenadas transformado

$h_{v}$ coeficiente métrico do sistema de coordenadas transformado

$H$ função definida

$J$ Jacobiano da transformação do sistema de coordenadas

$K$ fator de Hagenbach

$K_{d}$ fator de correção no fluxo do momentum

$K_{e}$ fator de correção de energia cinética

$K_{i}$ autofunções normalizadas relativas às autofunções $\psi_{i}$ [m]

$L_{h y}$ comprimento de entrada fluidodinâmico

$L_{h y}^{+} \quad$ comprimento de entrada fluidodinâmico adimensional

$M$ ordem de truncamento da expansão

$M_{j}$ norma das autofunções $\phi_{j}$

$N$ ordem de truncamento da expansão

$N_{i}$ norma das autofunções $\tilde{\bar{W}}_{i j}$

$p \quad$ pressão $[\mathrm{Pa}]$

Per perímetro do duto [m]

Po número de Poiseuille

Re número de Reynolds

$t$ tempo [s]

$u$ coordenada do sistema transformado

$u_{0}$ parâmetro que define contorno no plano $(u, v)$ $\boldsymbol{v} \quad$ vetor velocidade do fluido $[\mathrm{m} / \mathrm{s}]$

$v$ coordenada do sistema transformado

$v_{0}$ parâmetro que define contorno no plano $(u, v)$

$w$ componente axial da velocidade do fluido $[\mathrm{m} / \mathrm{s}]$

$w_{\text {máx }}$ velocidade máxima do fluido $[\mathrm{m} / \mathrm{s}]$

$w_{\text {méd }}$ velocidade média do fluido $[\mathrm{m} / \mathrm{s}]$

$W$ componente adimensional axial da velocidade

$W^{*}$ velocidade adimensional em termos da velocidade média

$W_{\text {máx }}$ velocidade adimensional máxima do fluido

$W_{\text {méd }}$ velocidade adimensional média do fluido

$\tilde{\bar{W}}_{i j}$ potencial velocidade adimensional transformado

$\tilde{\bar{W}}_{i j}$ potencial velocidade adimensional transformado

$x \quad$ coordenada espacial $[\mathrm{m}]$

$X \quad$ coordenada espacial adimensional

$y \quad$ coordenada espacial [m]

$Y \quad$ coordenada espacial adimensional

$z \quad$ coordenada espacial [m]

Z coordenada espacial adimensional

$Z_{j} \quad$ autofunções normalizadas relativas às autofunções $\phi_{j}$

\section{Letras Gregas}

$\phi_{j}$ autofunções relativa à coordenada $v$

$\lambda_{j}$ autovalores associados as autofunções $\psi_{i}$

$\Gamma \quad$ contorno dos dutos

$\mu \quad$ viscosidade dinâmica do fluido [Pa.s]

$\mu_{i} \quad$ autovalores associado a autofunção $\psi_{i}$

$\rho$ massa específica $\left[\mathrm{kg} / \mathrm{m}^{3}\right]$ 


\section{$\tau_{p, m e ́ d}$ tensão de cisalhamento média na parede
$\psi_{i} \quad$ autofunções relativas à coordenada $u$
$\zeta \quad$ variável complexa no plano $(u, v)$
$\omega \quad$ variável complexa no plano $(u, v)$ \\ $\Omega \quad$ domínio referente à área de seção transversal} $[\mathrm{Pa}]$

\section{Símbolos Especiais}

a Operador Nabla

o Operador Del

\section{Sobrescritos}

- Transformação Integral em relação à coordenada $u$

Transformação Integral em relação à coordenada $v$

\section{Subscritos}

$i$ Ordem do autovalor e de funções relacionadas

$j$ Ordem do autovalor e de funções relacionadas

\section{Introdução}

No processo de otimização de um trocador de calor os parâmetros termofluidodinâmicos são calculados visando à intensificação do coeficiente global de transferência de calor sem ultrapassar os limites admissíveis da perda de pressão (SHAH; LONDON, 1978). A determinação destes parâmetros envolve, basicamente, o cálculo do coeficiente de atrito e do número de Nusselt para os escoamentos interno e externo (KAKAÇ; SHAH; AUNG, 1998).

Uma nova concepção de trocadores de calor com dutos de seção bicônica, dispostos em um arranjo quincôncio, define uma estrutura de canais ondulados ao escoamento externo que permite um maior contato do fluido junto às paredes dos dutos e, consequentemente, uma diminuição dos efeitos dos vórtices na região posterior (ANTONINI ALVES, 2006).

Como primeiro passo desta proposta, neste trabalho é analisado o problema fluidodinâmico para o escoamento laminar desenvolvido de fluidos Newtonianos no interior de dutos de seção bicônica o qual se caracteriza pela difusão de momentum de forma similar ao processo de difusão de calor (MAIA et al., 2005; PELEGRINI et al., 2014).
A maior dificuldade para a obtenção de solução analítica deste problema consiste na impossibilidade da utilização da técnica de separação de variáveis e, também, pela dificuldade da aplicação das condições de contorno devido à característica bidimensional nãoconvencional da geometria bicônica (MAIA, 2003).

Diante destas razões, a Técnica da Transformada Integral Generalizada - TTIG (COTTA, 1998) é aplicada para a obtenção da solução da equação do momentum e uma mudança de coordenadas, através de uma Transformação Conforme apropriada, é utilizada para facilitar a aplicação das condições de contorno.

Os parâmetros fluidodinâmicos de interesse, tais como: velocidades média e máxima, fator de atrito de Fanning, número de Poiseuille, fator de Hagenbach e comprimento de entrada fluidodinâmico são, então, determinados e analisados para diversos formatos bicônicos.

\section{Procedimento Híbrido Analítico-Numérico}

Considera-se um escoamento laminar em dutos bicônicos. Desta forma, para fluidos Newtonianos com propriedades constantes, a equação do momentum para o sistema de coordenadas (Fig. 1), é escrita como: 


$$
\begin{aligned}
& \rho \frac{D v(x, y, z)}{D t}=-\nabla p(x, y, z)+\mu \nabla^{2} v(x, y, z) \\
& \{(x, y) \in \Omega, \quad z>0\}
\end{aligned}
$$

$$
\boldsymbol{v}(x, y, z)=0, \quad\{(x, y) \in \Gamma, \quad z>0\}
$$

sendo que, $\rho$ representa a massa específica do fluido, $\mu$ é a viscosidade dinâmica, $p$ o campo de pressão, $v$ o campo vetorial de velocidades, $\Gamma$ o contorno e $\Omega$ o domínio da geometria bicônica.

Figura 1 - Geometria original do problema.

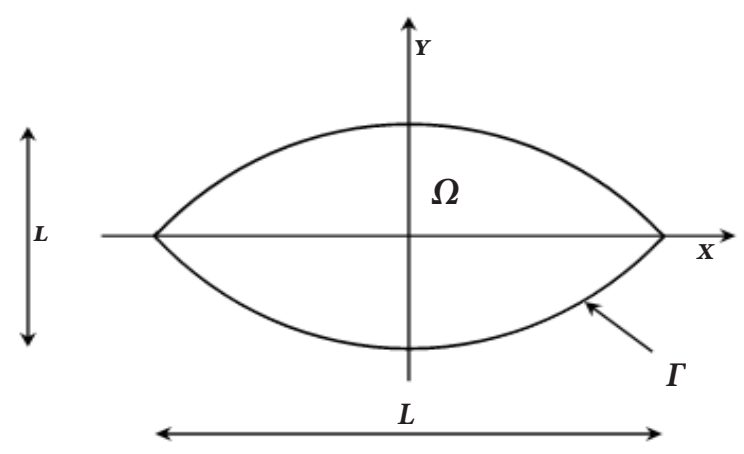

Fonte: o próprio autor.

Para escoamento desenvolvido e em regime permanente, a equação do momentum, Equação (1), se reduz a

$$
\begin{gathered}
\nabla^{2} \boldsymbol{v}(x, y)-\frac{1}{\mu} \nabla p(z)=0, \\
\{(x, y) \in \Omega, \quad z>0\}, \\
\boldsymbol{v}(x, y)=\mathbf{0}, \quad\{(x, y) \in \Gamma, \quad z>0\},
\end{gathered}
$$

sendo que,

$$
\begin{aligned}
& \boldsymbol{v}(x, y)=w(x, y) \boldsymbol{k} \\
& \nabla p(z)=\frac{d p(z)}{d z} \boldsymbol{k} .
\end{aligned}
$$

\section{Adimensionalização}

Reescrevendo a equação do momentum, Equação (3), na forma adimensional, tem-se que

$$
\begin{gathered}
\frac{\partial^{2} W(X, Y)}{\partial X^{2}}+\frac{\partial^{2} W(X, Y)}{\partial Y^{2}}+1=0, \\
\{(X, Y) \in \Omega, \quad Z>0\}, \\
W(X, Y)=0, \quad\{(X, Y) \in \Gamma, \quad Z>0\},
\end{gathered}
$$

com,

$$
\begin{aligned}
& X=\frac{x}{D_{h}}, \\
& Z=\frac{z}{D_{h}}, \\
& Z=\frac{z}{D_{h}},
\end{aligned}
$$

$$
D_{h}=\frac{4 A_{S}}{P e r},
$$

$$
W(X, Y)=\frac{w(X, Y)}{\left[\frac{D_{h}}{\mu}\left(-\frac{d p(Z)}{d Z}\right)\right]},
$$

sendo, $D_{h}$ o diâmetro hidráulico, $A_{S}$ a área da seção transversal, Per o perímetro do contorno, $w$ a velocidade do fluido Newtoniano e $W$ velocidade adimensional. Desta maneira, a equação do momentum, Equação (7), se apresenta na forma da equação de Poisson para o potencial $W(X, Y)$.

\section{Transformação de Coordenadas}

Para facilitar o tratamento analítico, foi utilizada uma Transformação Conforme expressa por

$$
\zeta=\frac{1}{2} i \bar{\omega}^{2}
$$


sendo que, $\zeta$ é a variável complexa no plano $\zeta$ $=X+i Y$ e $\omega$ é o conjugado da variável complexa no plano transformado $\omega=u+i v$. Esta relação permite transformar o domínio bicônico no plano
$(X, Y)$ em um domínio retangular no plano $(u, v)$, limitado pelas retas $u=0, u=-u_{0}, u=u_{0}, v=0$, $v=-v_{0}, v=v_{0}$, com $u_{0}=v_{0}$, conforme ilustrado na Fig. 2.

Figura 2 - Transformação do domínio bicônico $\left(u_{0} / v_{0}=1,00\right)$ no plano $(X, Y)$ para o plano $(u, v)$.

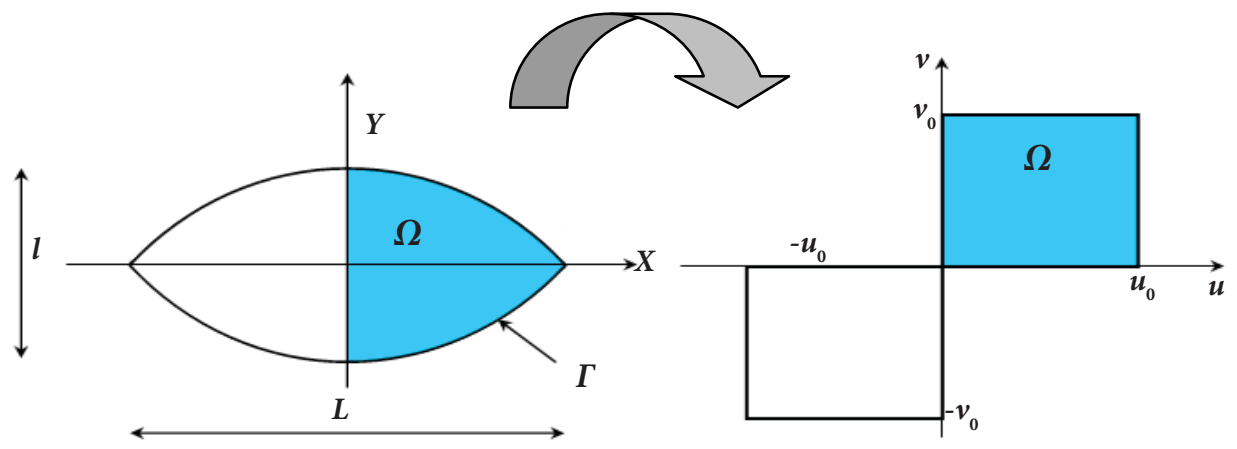

Fonte: o próprio autor.

As relações de transformação de coordenadas são

$$
\begin{aligned}
& Y=\frac{1}{2}\left(u^{2}-v^{2}\right) \\
& Z=z \\
& Y=\frac{1}{2}\left(u^{2}-v^{2}\right)
\end{aligned}
$$

A Transformação Conforme dada pela Equação (14) satisfaz as condições de Cauchy-Riemann. Portanto, os coeficientes métricos $h_{u}, h_{v}$ e o Jacobiano $J(u, v)$ da transformação são expressos por:

$$
\begin{aligned}
& h_{u}(u, v)=h_{v}(u, v)=\sqrt{\left(u^{2}+v^{2}\right)} \\
& J(u, v)=\frac{\partial(X, Y)}{\partial(u, v)}=\left(u^{2}+v^{2}\right)
\end{aligned}
$$

Este novo sistema de coordenadas é ortogonal e gera parábolas confocais de eixo comum (SPIELGEL; LIU, 1999), aqui denominadas simplesmente por geometrias bicônicas (Fig. 3).

Figura 3 - Transformação do domínio bicônico no plano $(X, Y)$ para o plano $(u, v)$.

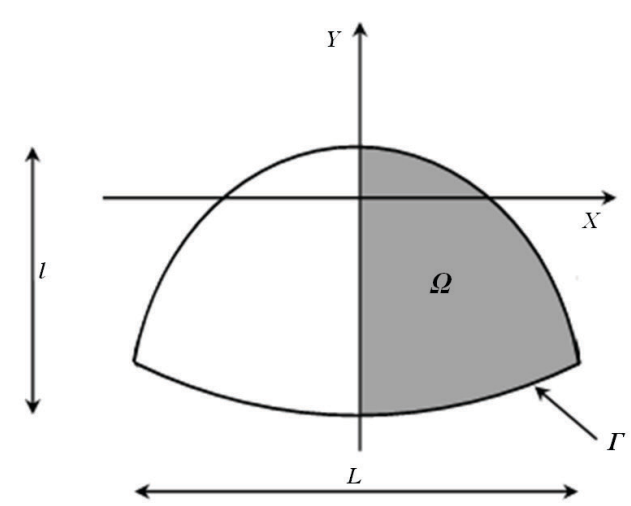

(a) $u_{0} / v_{0}=0,50$

Fonte: o próprio autor.

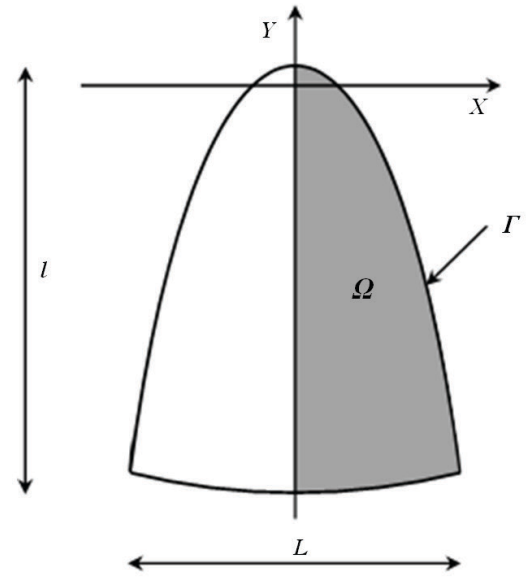

(b) $u_{0} / v_{0}=0,20$ 
Com estas novas variáveis definidas, a equação do momentum, transforma-se em

$$
\begin{gathered}
\frac{\partial^{2} W(u, v)}{\partial u^{2}}+\frac{\partial^{2} W(u, v)}{\partial v^{2}}=H(u, v), \\
\{(u, v) \in \Omega\}, \\
W(u, v)=0 \quad\{(u, v) \in \Gamma, \quad z>0\} \\
\operatorname{com} H(u, v)=-J(u, v) .
\end{gathered}
$$

No plano $(u, v)$ é suficiente considerar somente o domínio definido na região $\left\{0 \leq u \leq u_{0}\right\}$ e $\left\{0 \leq v \leq v_{0}\right\}$ (região sombreada $\Omega$ ).

As condições de contorno no novo sistema de coordenadas são expressas por

$$
\begin{aligned}
& \frac{\partial W(u, v)}{\partial u}=0, \quad\left\{u=0, \quad 0 \leq v \leq v_{0}\right\}, \\
& \frac{\partial W(u, v)}{\partial u}=0, \quad\left\{u=u_{0}, \quad 0 \leq v \leq v_{0}\right\}, \\
& \frac{\partial W(u, v)}{\partial v}=0, \quad\left\{0 \leq u \leq u_{0}, \quad v=0\right\}, \\
& W(u, v)=0, \quad\left\{0 \leq u \leq u_{0}, \quad v=v_{0}\right\},
\end{aligned}
$$

Os autovalores e as autofunções normalizadas associados a este problema são dados, respectivamente, por

$$
\begin{gathered}
\mu_{i}=\frac{(i-1) \pi}{u_{0}}, \quad i=1,2,3 \ldots \\
\psi_{i}(u)=\cos \left(\mu_{i} u\right) .
\end{gathered}
$$

Estas autofunções permitem o desenvolvimento do par transformada-inversa

$$
\begin{gathered}
\bar{W}_{i}(v)=\int_{0}^{u_{0}} K_{i}(u) W(u, v) d u \\
W(u, v)=\sum_{i=1}^{\infty} K_{i}(u) \bar{W}_{i}(v) .
\end{gathered}
$$

sendo que, $\bar{W}_{i}(v)$ é o potencial transformado em $u$ e $K_{i}(u)$ são as autofunções normalizadas, dadas por

\section{Técnica da Transformada Generalizada (TTIG)}

Para a obtenção da solução da equação do momentum no novo sistema de coordenadas aplicase a Técnica da Transformada Integral Generalizada - TTIG sobre a Equação (20) visando proceder a remoção das derivadas de segunda ordem. Para este fim, escreve-se o potencial $W(u, v)$ em termos de uma expansão em autofunções normalizadas obtidas de problemas auxiliares de autovalor para cada coordenada espacial. Neste sentido, considere, primeiramente, o problema auxiliar de autovalor,

$$
\frac{d^{2} \psi(u)}{d u^{2}}+\mu^{2} \psi(u)=0, \quad\left\{0 \leq u \leq u_{0}\right\},
$$

com,

$$
K_{i}(u)=\frac{\psi_{i}(u)}{\sqrt{N_{i}}},
$$

com,

$$
N_{i}=\int_{0}^{u_{0}} \psi_{i}^{2}(u) d u=\left\{\begin{array}{cc}
u_{0}, & i=1, \\
u_{0} / 2, & i \neq 1 .
\end{array}\right.
$$

sendo que, $N_{i}$ são as integrais de normalização das autofunções $\psi_{\mathrm{i}}(u)$.

Efetuando-se o produto interno das autofunções normalizadas $K_{i}(u)$ com a equação do momentum e fazendo uso das condições de contorno e das equações que definem o problema auxiliar de autovalor, obtém-se a primeira transformação da equação diferencial, 


$$
\begin{gathered}
A_{i}(v)+\mu_{i}^{2} \bar{W}_{i}(v)=\frac{\partial^{2} \bar{W}_{i}(v)}{\partial v^{2}}, \quad i=1,2,3 \ldots \\
A_{i}(v)=\int_{0}^{u_{0}} K_{i}(u) H(u, v) d u
\end{gathered}
$$

Para proceder a transformação integral relativo à coordenada $v$, considera-se agora o seguinte problema de autovalor,

$$
\frac{d^{2} \phi(v)}{d v^{2}}+\lambda^{2} \phi(v)=0, \quad\left\{0 \leq v \leq v_{0}\right\}
$$

com,

$$
\begin{gathered}
\frac{d \phi(0)}{d v}=0, \\
\phi\left(v_{0}\right)=0 .
\end{gathered}
$$

Os autovalores e as autofunções normalizadas para este novo problema são dadas por

$$
\begin{gathered}
\lambda_{j}=\frac{(2 j-1) \pi}{2 v_{0}}, \quad j=1,2,3 \ldots \\
\phi_{j}(v)=\cos \left(\lambda_{j} v\right) .
\end{gathered}
$$

Estas autofunções permitem o desenvolvimento do seguinte par transformada-inversa

$$
\begin{gathered}
\tilde{\bar{W}}_{i j}=\int_{0}^{v_{0}} \int_{0}^{u_{0}} K_{i}(u) Z_{j}(v) W(u, v) d u d v, \\
W(u, v)=\sum_{i=0}^{\infty} \sum_{j=1}^{\infty} K_{i}(u) Z_{j}(v) \tilde{\bar{W}}_{i j}(Z) .
\end{gathered}
$$

sendo que, $Z_{j}(v)$ são as autofunções normalizadas e são expressas por

$$
\begin{aligned}
& Z_{j}(v)=\frac{\phi_{j}(v)}{\sqrt{M_{j}}} \\
& M_{j}=\int_{0}^{v_{0}} \phi_{j}^{2}(v) d v=\frac{v_{0}}{2},
\end{aligned}
$$

sendo que, $M_{j}$ são as integrais de normalização das autofunções $\phi_{i}(v)$.

Efetuando-se o produto interno das autofunções normalizadas $Z_{j}(v)$ com a equação diferencial transformada em $u$ e fazendo uso das condições de contorno e das relações de ortogonalidade relativas ao segundo problema de autovalor, obtém-se a transformação integral da equação do momentum,

$$
\begin{gathered}
B_{i j}+\left(\mu_{i}^{2}+\lambda_{j}^{2}\right) \tilde{\overline{\bar{W}}}_{i j}=0, \quad i, j=1,2,3 \ldots \\
B_{i j}=\int_{0}^{v_{0}} Z_{j}(v) A_{i}(v) d v= \\
=\int_{0}^{v_{0}} \int_{0}^{u_{0}} K_{i}(u) Z_{j}(v) H(u, v) d u d v .
\end{gathered}
$$

Observa-se que os parâmetros $B_{i j}$ são integráveis e que o sistema dado pela Equação (46) é algébrico, linear e desacoplado. Portanto, o potencial transformado pode ser obtido de forma direta,

$$
\tilde{\bar{W}}_{i j}=-\frac{B_{i j}}{\mu_{i}^{2}+\lambda_{j}^{2}},
$$

permitindo, assim, a obtenção da solução analítica do potencial velocidade adimensional,

$$
\begin{gathered}
W(u, v)=\sum_{i=1}^{\infty} \sum_{j=1}^{\infty} K_{i}(u) Z_{j}(v) \tilde{\bar{W}}_{i j}= \\
=-\sum_{i=1}^{\infty} \sum_{j=1}^{\infty} \frac{B_{i j}}{\mu_{i}^{2}+\lambda_{j}^{2}} K_{i}(u) Z_{j}(v) .
\end{gathered}
$$

Para fins computacionais, o potencial transformado pode ser determinado numericamente quando a expansão é truncada em uma dada ordem 
$i=M$ e $j=N$. Portanto, utilizando-se da fórmula de inversão, determina-se o potencial velocidade adimensional por

$$
\begin{gathered}
W(u, v)=\sum_{i=1}^{M} \sum_{j=1}^{N} K_{i}(u) Z_{j}(v) \tilde{\bar{W}}_{i j}= \\
=-\sum_{i=1}^{M} \sum_{j=1}^{N} \frac{B_{i j}}{\mu_{i}^{2}+\lambda_{j}^{2}} K_{i}(u) Z_{j}(v) .
\end{gathered}
$$

Obviamente, quanto maior $N$ e $M$ maior será a precisão dos resultados, sendo que, do ponto de vista computacional, o duplo somatório poderia ser resolvido como proposto por Mikhailov e Cotta (1996), permitindo a redução deste custo através do reordenamento dos termos e substituição por um somatório simples. No entanto, pela simplicidade do problema em questão, isso não se faz necessário.

Da definição da velocidade $W(u, v)$, determinase, então, o campo de velocidade para o escoamento laminar completamente desenvolvido em dutos bicônicos.

\section{Parâmetros Fluidodinâmicos de Interesse}

\section{Velocidade Adimensional}

A velocidade média adimensional pode ser expressa por

$$
W_{\text {med }}=\frac{1}{A_{S}} \int_{A_{S}} W(X, Y) d A_{S}
$$

Dessa forma, a velocidade adimensional $W^{*}(X, Y)$ definida em termos da velocidade média, pode ser determinada por

$$
W^{*}(X, Y)=\frac{w(X, Y)}{w_{\text {med }}(X, Y)}=\frac{W(X, Y)}{W_{\text {med }}(X, Y)},
$$

A velocidade adimensional máxima $W_{\text {max }}^{*}$ definida em termos da velocidade média, é expressa por
$W_{\text {máx }}^{*}=\frac{w_{\text {máx }}}{w_{\text {méd }}(X, Y)}=\frac{W_{\text {máx }}}{W_{\text {méd }}(X, Y)}$,

sendo que, $w_{\text {máx }}$ e $W_{\text {máx }}$ são os valores máximos da velocidade e da velocidade adimensional, respectivamente.

\section{Fator de Atrito de Fanning}

$\mathrm{O}$ fator de atrito de Fanning, $f$, também conhecido como coeficiente de atrito, é definido por

$$
f=\frac{2 \tau_{p, \text { méd }}}{\rho w_{\text {méd }}^{2}}
$$

sendo que, $\tau_{p \text {,méd }}$ é a tensão de cisalhamento média na parede e é expressa por

$$
\tau_{p, \text { méd }}=-\frac{d p}{d z}\left(\frac{A_{S}}{P e r}\right)
$$

\section{Número de Poiseuille}

Para escoamento laminar desenvolvido no interior de dutos o produto $(f R e)$ é igual a uma constante. Das relações de adimensionalização, obtém-se que

$$
f R e=P o=\frac{1}{2 W_{\text {med }}} .
$$

\section{Fator de Hagenbach}

O número do incremento na queda de pressão ou fator de Hagenbach, $K(\infty)$, de acordo com (LUNDGREN; SPARROW; STARR, 1964), é definido como

$$
K(\infty)=\frac{2}{A_{S}} \int_{A_{S}}\left\{\left[\frac{w(u, v)}{w_{\text {méd }}}\right]^{3}-\left[\frac{w(u, v)}{w_{\text {méd }}}\right]^{2}\right\} d A_{S}
$$


ou,

$$
K(\infty)=2\left[K_{e}(\infty)-K_{d}(\infty)\right]
$$

sendo que, $K_{e}(\infty)$ e $K_{d}(\infty)$, são respectivamente, o fator de correção de energia cinética e o fator de correção no fluxo de momentum, expressos por

$$
\begin{aligned}
& K_{e}(\infty)=\frac{1}{A_{S}} \int_{A_{S}}\left[\frac{w(u, v)}{w_{\text {méd }}}\right]^{3} d A_{S}, \\
& K_{d}(\infty)=\frac{1}{A_{S}} \int_{A_{S}}\left[\frac{w(u, v)}{w_{\text {méd }}}\right]^{2} d A_{S} .
\end{aligned}
$$

\section{Comprimento de Entrada Fluidodinâmico}

O comprimento de entrada fluidodinâmico, $L_{h y}$, é definido, com sendo a posição em que a velocidade máxima atinge $99 \%$ da velocidade correspondente à região fluidodinamicamente desenvolvida quando o escoamento de entrada é uniforme (SHAH; LONDON, 1978). O comprimento de entrada fluidodinâmico adimensional, de acordo com (MCCOMAS, 1967) pode ser expresso por

$$
L_{h y}^{+}=\frac{L_{h y}}{D_{h} \operatorname{Re}}=\frac{\left(W_{\text {max }}^{*}\right)^{2}-1-K(\infty)}{4 f \operatorname{Re}}
$$

\section{Resultados e Discussão}

Para a obtenção dos resultados numéricos, o Método de Quadratura de Gauss foi utilizado para o cálculo das integrais envolvidas nos coeficientes $B_{i j}$ e demais parâmetros físicos de interesse. Desta forma, foi necessária, também, a determinação das autofunções e do Jacobiano nos pontos de quadratura. Feito isso, o sistema de equações algébrico, linear e desacoplado foi resolvido para a determinação dos coeficientes do potencial transformado fazendo uso da rotina DIVPAG da biblioteca IMSL Fortran (1994) e do truncamento da expansão dada pela Equação (50) para diversas ordens $M$ e $N$.
Foi verificado através de uma análise de convergência que com a utilização de até 50 termos na expansão da série em cada coordenada foi possível à obtenção de três casas decimais de precisão na região de convergência mais lenta $\left(u_{0}\right)$ $v_{0} \leq 0,20$ ), enquanto que nas demais regiões obtémse a mesma precisão com uma ordem $N=M=40$ de truncamento na série, ou menos.

O tempo de processamento gasto no cômputo dos parâmetros fluidodinâmicos de interesse foi pequeno, cerca de 5 a 10 segundos em um computador pessoal comum (processador Intel Core $^{T M} i 7$ 3,6GHz com 16GB de memória RAM).

$\mathrm{Na}$ Figura 4 são ilustrados os perfis de velocidade e os mapas de isovelocidades do escoamento laminar de fluidos Newtonianos considerando dutos bicônicos com diferentes configurações geométricas $\left(u_{0} / v_{0}=0,10,0,50\right.$ e 1,00). Como esperado, a velocidade na parede é nula (condição de nãodeslizamento) enquanto que a velocidade máxima é encontrada na região próxima ao centro do duto.

Figura 4 - Perfis de velocidades para o escoamento em dutos bicônicos.

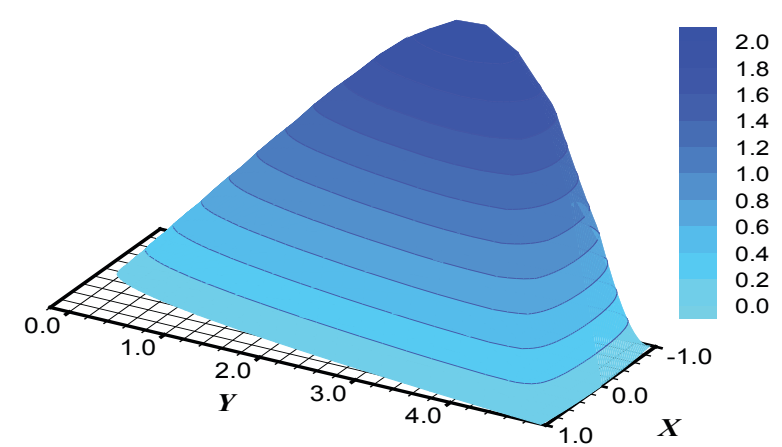

(a) perfil de velocidade tridimensional para $u_{0} / v_{0}=0,10$ Fonte: o próprio autor.

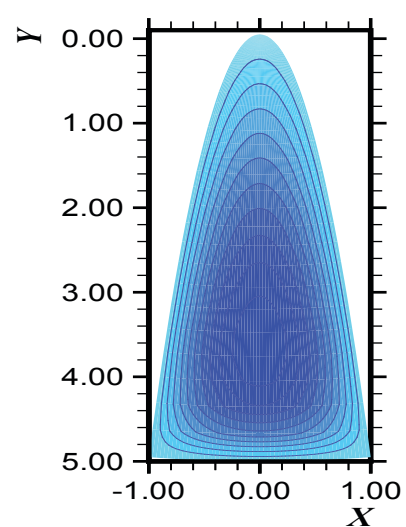

(b) mapa de isovelocidades para $\underset{u_{0}}{\boldsymbol{X}} / v_{0}=0,10$

Fonte: o próprio autor. 


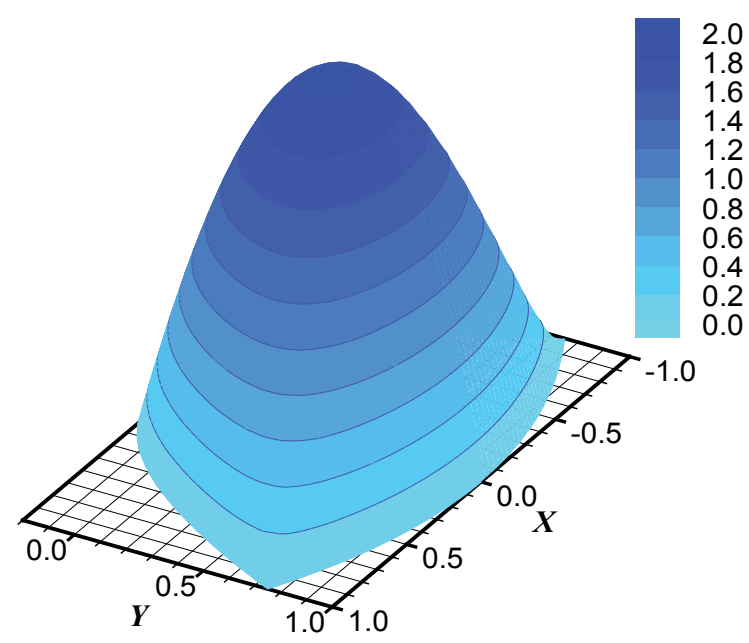

(c) perfil de velocidades tridimensional para $u_{0} / v_{0}=0,50$ Fonte: o próprio autor.

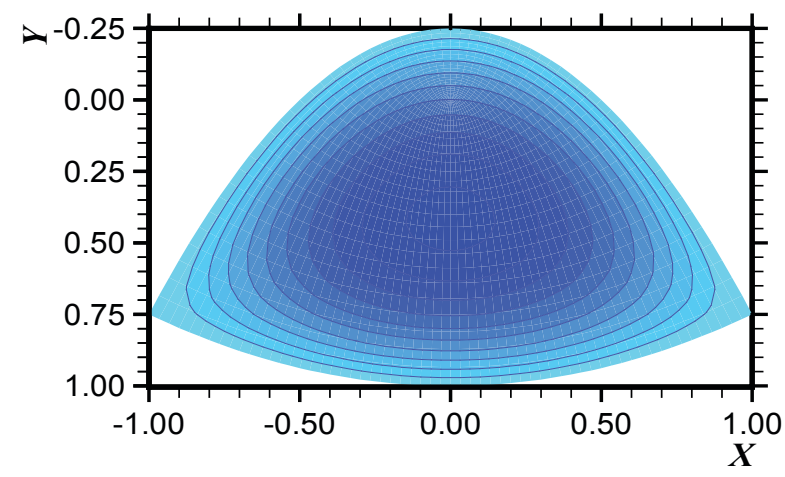

(d) mapa de isovelocidades para $u_{0} / v_{0}=0,50$

Fonte: o próprio autor.

Na Tabela 1 e nas Figuras 5 a 8 são apresentados os resultados encontrados para a velocidade adimensional máxima, o número de Poiseuille, o fator de Hagenbach e o comprimento de entrada fluidodinâmico adimensional considerando diversas configurações geométricas dos dutos bicônicos.

Nota-se que estes parâmetros fluidodinâmicos de interesse diminuem com o aumento da razão

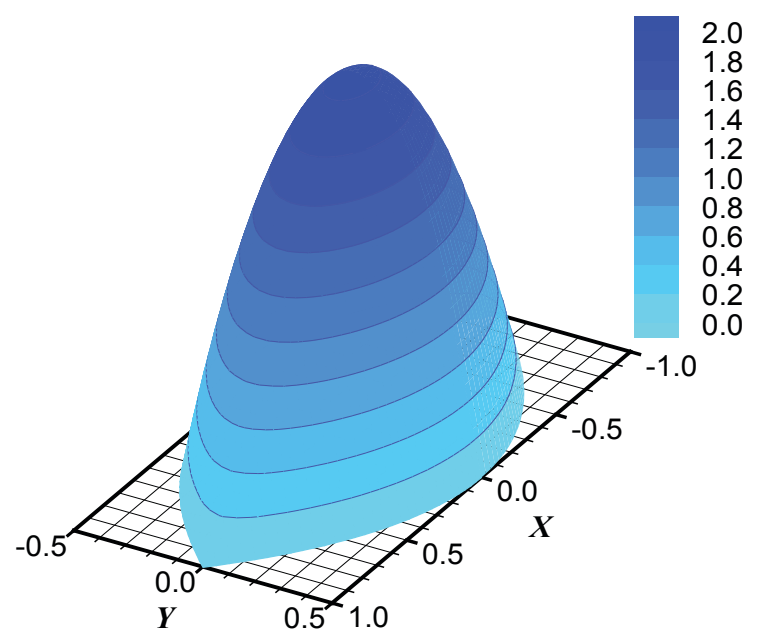

(e) perfil de velocidades tridimensional para $u_{0} / v_{0}=1,00$ Fonte: o próprio autor.

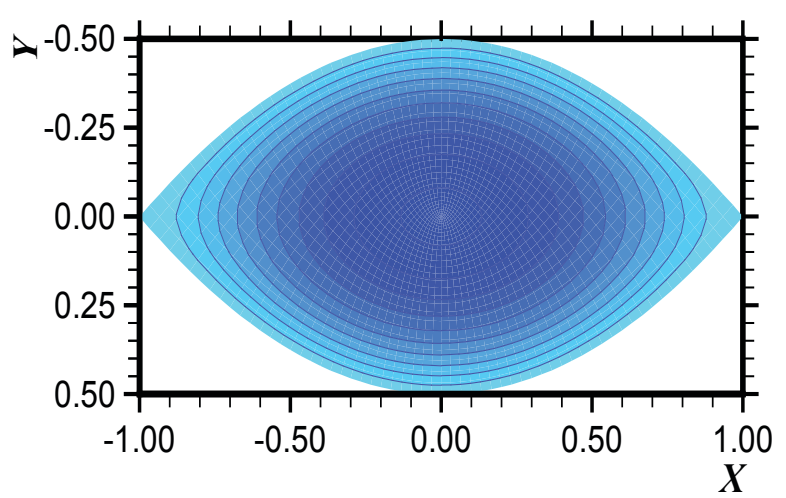

(f) mapa de isovelocidades para $u_{0} / v_{0}=1,00$

Fonte: o próprio autor.

$u_{0} / v_{0}$ e que convergem para $W_{\text {max }}=2,056 ; f R e=$ 15,$52 ; K(\infty)=1,455$ e $L_{h y}{ }^{+}=0,02856$; quando $u_{0}{ }^{\prime}$ $v_{0} \rightarrow 1$. Destaca-se ainda que o menor valor do número de Poiseuille, $f R e=15,08$, é encontrado quando $u_{0} / v_{0} \approx 0,35$ - geometricamente são os dutos que mais se assemelham aos dutos circulares $\left(f R e_{\text {circular }}=16\right)(\mathrm{SHAH} ; \mathrm{LONDON}$, 1978). 
Tabela 1 - Resultados dos parâmetros fluidodinâmicos para diversas configurações geométricas dos dutos bicônicos.

\begin{tabular}{|c|c|c|c|c|}
\hline$u_{0} / v_{0}$ & $W_{\text {máx }}^{*}$ & $f R e$ & $K(\infty)$ & $L_{\boldsymbol{g}}^{+}$ \\
\hline 0,05 & 2,2507 & 16,960 & 1,7407 & 0,03427 \\
\hline 0,10 & 2,1678 & 16,254 & 1,6324 & 0,03179 \\
\hline 0,15 & 2,1256 & 15,754 & 1,5699 & 0,03092 \\
\hline 0,20 & 2,1036 & 15,386 & 1,5355 & 0,03070 \\
\hline 0,25 & 2,0915 & 15,193 & 1,5165 & 0,03057 \\
\hline 0,30 & 2,0847 & 15,104 & 1,5051 & 0,03047 \\
\hline 0,35 & 2,0796 & 15,084 & 1,4970 & 0,03029 \\
\hline 0,40 & 2,0751 & 15,107 & 1,4903 & 0,03005 \\
\hline 0,45 & 2,0724 & 15,153 & 1,4843 & 0,02987 \\
\hline 0,50 & 2,0693 & 15,210 & 1,4789 & 0,02964 \\
\hline 0,55 & 2,0667 & 15,269 & 1,4740 & 0,02943 \\
\hline 0,60 & 2,0642 & 15,325 & 1,4696 & 0,02922 \\
\hline 0,65 & 2,0622 & 15,375 & 1,4658 & 0,02905 \\
\hline 0,70 & 2,0605 & 15,418 & 1,4627 & 0,02891 \\
\hline 0,75 & 2,0591 & 15,453 & 1,4601 & 0,02879 \\
\hline 0,80 & 2,0580 & 15,480 & 1,4581 & 0,02870 \\
\hline 0,85 & 2,0572 & 15,501 & 1,4567 & 0,02863 \\
\hline 0,90 & 2,0567 & 15,514 & 1,4557 & 0,02859 \\
\hline 0,95 & 2,0564 & 15,522 & 1,4552 & 0,02857 \\
\hline 1,00 & 2,0563 & 15,524 & 1,4550 & 0,02856 \\
\hline
\end{tabular}

Fonte: o próprio autor.

Fioura 5 - Velocidade adimensional máxima em

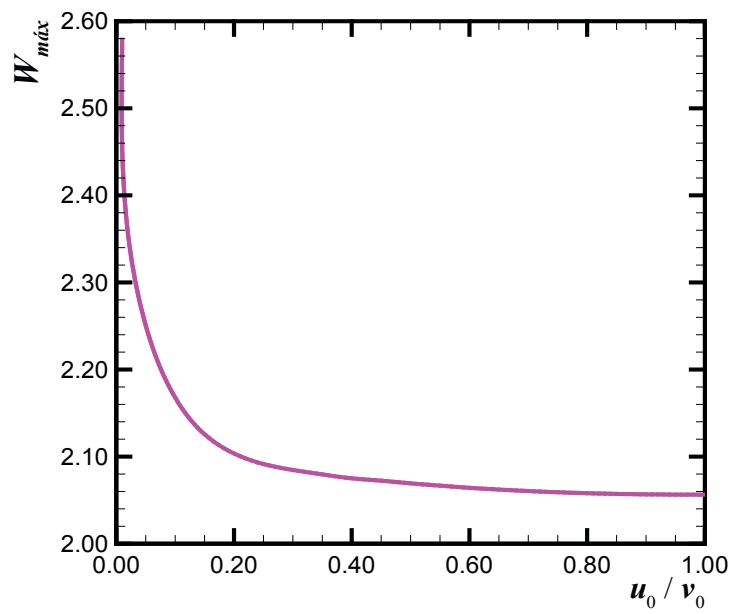

Fonte: o próprio autor.
Finalmente, ressalta-se que para este problema de escoamento laminar no interior de dutos bicônicos nenhum resultado foi encontrado na literatura consultada.

Figura 6 - Número de Poiseuille em dutos bicônicos.

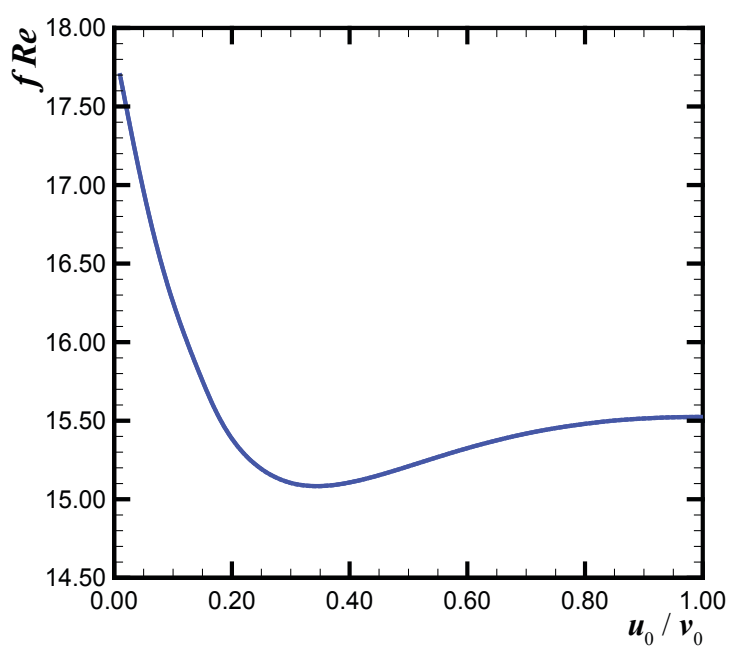

Fonte: o próprio autor.

Figura 7 - Fator de Hagenbach em dutos bicônicos.

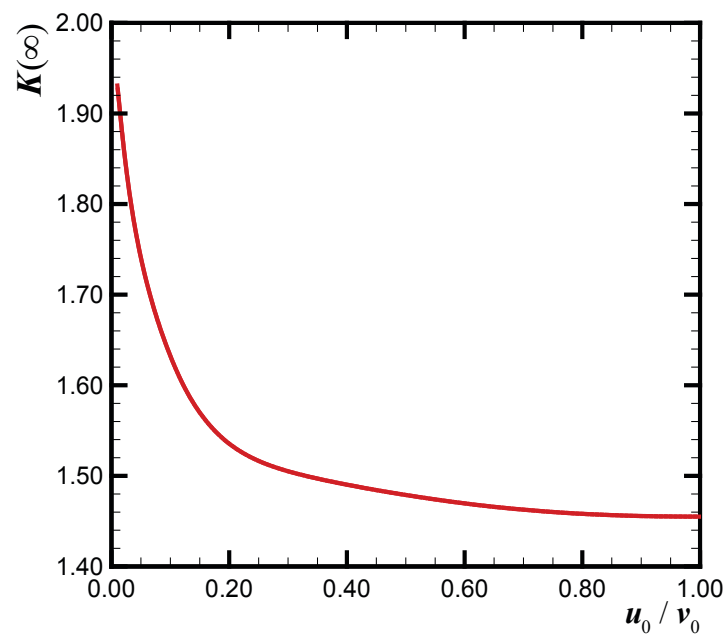

Fonte: o próprio autor. 
Figura 8 - Comprimento de entrada fluidodinâmico adimensional em dutos bicônicos.

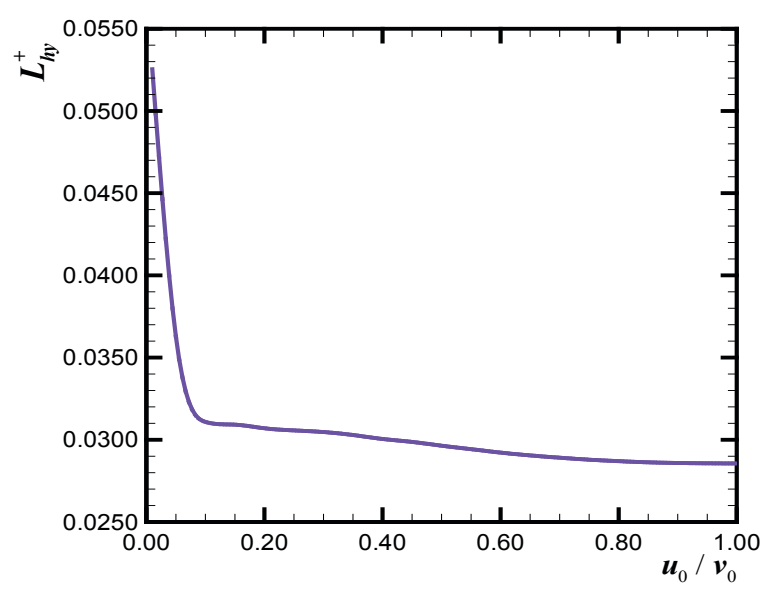

Fonte: o próprio autor.

\section{Conclusões}

No presente trabalho foi apresentada uma solução híbrida analítico-numérica do problema fluidodinâmico de escoamento laminar de fluidos Newtonianos em dutos bicônicos utilizando a Técnica da Transformada Integral Generalizada TTIG e a uma Transformação Conforme.

Os parâmetros fluidodinâmicos de interesse, tais como: velocidades média e máxima, fator de atrito de Fanning, número de Poiseuille, fator de Hagenbach e comprimento de entrada fluidodinâmico foram obtidos para dutos de configurações geométricas bicônicas. Estes resultados são inéditos e serão utilizados para uma nova concepção de trocadores de calor com dutos bicônicos, dispostos em um arranjo quincôncio, visando o processo de intensificação do coeficiente global de transferência de calor sem ultrapassar os limites admissíveis da perda de pressão.

Finalmente, observa-se que a Técnica da Transformada Integral Generalizada (TTIG) está sendo aplicada com sucesso na obtenção de solução de problemas difusivos e difusivo-convectivos multidimensionais, os quais muitas vezes não admite solução pelas técnicas analíticas clássicas.

\section{Referências}

ANTONINI ALVES, T. Aplicação da Transformada Integral e da Transformação Conforme na solução de uma classe de problemas difusivo-convectivos em domínios de geometrias não-convencionais. 2006. Dissertação (Mestrado em Engenharia Mecânica) Departamento de Engenharia Mecânica, Faculdade de Engenharia de Ilha Solteira da Universidade Estadual Paulista "Júlio de Mesquita Filho".

COTTA, R. M. The integral transform method in thermal and fluids science and engineering. New York, USA: Begell House, 1998.

IMSL LIBRARY. GNB Building. Texas, USA: Edition 7, 7500 Bellaire Blvd, 77036, 1994.

KAKAÇ, S.; SHAH, R. K.; AUNG, W. Handbook of single-phase convective heat transfer. New York, USA: John Wiley, 1998.

LUNDGREN, T. S.; SPARROW, E. M.; STARR, J. B. Pressure drop due to the entrance region in ducts of arbitrary cross section. Journal of Basic Engineering, v. 86, p. 620-626, 1964.

MAIA, C. R. M. Solução de problemas difusivos e difusivos: convectivos em domínio de geometria elíptica e bicôncava pela técnica da transformada integral generalizada. 2003. Tese (Doutorado em Engenharia Mecânica) - Departamento de Energia, Faculdade de Engenharia Mecânica da Universidade Estadual de Campinas.

MAIA, C. R. M.; RAMOS, R. A. V.; PELEGRINI, M. F.; ANTONINI ALVES, T. Heat transfer study in slug flow on elliptical ducts cross section by Generalized Integral Transform Technique. Engenharia Térmica, v. 4, n. 2, p. 154-163, 2005.

MIKHAILOV, M. D.; COTTA, R. M. Ordering rules for double or triple eigenseries in the solution of multidimensional heat and fluid flow problems. International Communications in Heat and Mass Transfer, v. 23, p. 299-303, 1996.

McCOMAS, S. T. Hydrodynamic entrance lengths for ducts of arbitrary cross section. Journal of Basic Engineering, v. 89, p. 847-850, 1967. 
PELEGRINI, M. F.; ANTONINI ALVES, T.; NISHIDA, F. B.; RAMOS, R. A. V.; MAIA, C. R. M.; Hybrid analytical-numerical analysis of SAE 4150 alloy steel rods cooling. Advanced Materials Research (Online), v. 1082, p. 187-190, 2014.

SHAH, R. K.; LONDON, A. L. Laminar flow forced convection in ducts. New York, USA: Academic Press, 1978.

SPIEGEL, M. R.; LIU, J. Mathematical handbook of formulas and tables. New York, USA: McGraw-Hill, 1999. 
